Yahya Shehabi

Lucy Chan

Suhaini Kadiman

Anita Alias

Wan Nasrudin Ismail

Mohd Ali T. Ismail Tan

Tien Meng Khoo

Saedah Binti Ali

Mat Ariffin Saman

Ahmad Shaltut

Cheng Cheng Tan

Cow Yen Yong

Michael Bailey

The Sedation Practice in Intensive Care

Evaluation (SPICE) Study Group investigators

\title{
Sedation depth and long-term mortality in mechanically ventilated critically ill adults: a prospective longitudinal multicentre cohort study
}

Received: 4 August 2012

Accepted: 22 December 2012

Published online: 24 January 2013

(C) The Author(s) 2013. This article is published with open access at

Springerlink.com

Participating centers and co-investigators are listed in the Appendix.

\section{Y. Shehabi ( $)$}

Department of Intensive Care, University

New South Wales Clinical School,

The Prince of Wales Hospital, Barker St.,

Randwick, NSW 2031, Australia

e-mail: y.shehabi@unsw.edu.au

Tel.: +61-2-93824721

Fax: +61-2-93824870

L. Chan

Department of Anesthesiology,

Faculty of Medicine, University of Malaya,

Lembah Pantai, 50603 Kuala Lumpur,

Malaysia

S. Kadiman

National Heart Institute (Institut Jantung

Negara), 145 Jalan Tun Razak,

50400 Kuala Lumpur, Malaysia

\section{A. Alias}

Department of Anesthesia and Intensive

Care, Hospital Melaka, Jalan Mufti $\mathrm{Hj}$

Khalil, 75400 Melaka, Malaysia

W. N. Ismail

Hospital Raja Perempuan Zainab II Kota

Bahru-HKB, Kelantan, Malaysia

\section{A. T. I. Tan}

Department of Anesthesia and Intensive

Care, General ICU, Kuala Lumpur General

Hospital, Jalan Pahang, 50586 Kuala

Lumpur, Malaysia

\section{T. M. Khoo}

Department of Anesthesiology and Intensive Care, Hospital Queen Elizabeth, 88586 Kota Kinabalu, Sabah, Malaysia

\section{S. B. Ali}

Department of Anesthesiology, School of Medicine, Hospital University Sains Malaysia, Health Campus,

16150 Kota Bahru, Kelantan, Malaysia

M. A. Saman

Department of Anesthesiology and Intensive Care, Sarawak General Hospital, Jalan Hospital, 93586 Kuching,

Sarawak, Malaysia

A. Shaltut

Hospital Sultanah Bahiyah, Alor Setar,

HAS, Kedah, Malaysia

C. C. Tan

ICU Selatan, 2nd Floor, Hospital Sultanah Aminah, Jalan Persiaran Abu Bakar, Johor Bahru, Johor, Malaysia

C. Y. Yong

Penang General Hospital, PGH, Penang, Malaysia

\section{Bailey}

Australian New Zealand Intensive Care Research Centre, School of Public Health and Preventive Medicine Monash University, Melbourne, Australia
Y. Shehabi

University New South Wales,

Prince of Wales Hospital,

Sydney, Australia

Abstract Purpose: To ascertain the relationship among early (first $48 \mathrm{~h}$ ) deep sedation, time to extubation, delirium and long-term mortality. Methods: We conducted a multicentre prospective longitudinal cohort study in 11 Malaysian hospitals including medical/surgical patients ( $n=259$ ) who were sedated and ventilated $\geq 24 \mathrm{~h}$. Patients were followed from ICU admission up to 28 days in ICU with 4-hourly sedation and daily delirium assessments and 180-day mortality. Deep sedation was defined as Richmond Agitation Sedation Score (RASS) $\leq-3$.

Results: The cohort had a mean (SD) age of 53.1 (15.9) years and APACHE II score of 21.3 (8.2) with hospital and 180-day mortality of 82 $(31.7 \%)$ and 110/237 (46.4\%). Patients were followed for 2,657 ICU days and underwent 13,836 RASS assessments. Midazolam prescription was predominant compared to propofol, given to $241(93 \%)$ versus 72 (28\%) patients $(P<0.0001)$ for 966 $(39.6 \%)$ versus $183(7.5 \%)$ study days respectively. Deep sedation occurred in (182/257) $71 \%$ patients at first assessment and in $159(61 \%)$ 
patients and 1,658 (59\%) of all RASS assessments at $48 \mathrm{~h}$. Multivariable Cox proportional hazard regression analysis adjusting for a priori assigned covariates including sedative agents, diagnosis, age,

APACHE II score, operative, elective, vasopressors and dialysis showed that early deep sedation was independently associated with longer time to extubation [hazard ratio (HR)
0.93, $95 \%$ confidence interval $(\mathrm{CI})$ $0.89-0.97, P=0.003]$, hospital death (HR 1.11, $95 \%$ CI 1.05-1.18, $P<0.001)$ and 180 -day mortality (HR 1.09, $95 \%$ CI 1.04-1.15, $P=0.002$ ), but not time to delirium (HR 0.98, $P=0.23$ ). Delirium occurred in 114 (44\%) of patients. Conclusion: Irrespective of sedative choice, early deep sedation was independently associated with delayed extubation and higher mortality, and thus was a potentially modifiable risk in interventional trials.

Keywords Sedation depth . Mechanical ventilation - Delirium . Critically ill $\cdot$ Mortality

\section{Introduction}

Sedation is an integral part of intensive care practice to minimise patient discomfort and anxiety, facilitate mechanical ventilation and allow essential intensive care procedures [1, 2]. Complications associated with sedative prescription and in particular deep sedation with adverse outcomes have been well described [3, 4]. Reports of shorter ventilation time, shorter ICU stay and attenuation of delirium with protocols targeting light sedation [5-8] highlight the need to avoid deep sedation proactively [9].

There are no reliable data on the compliance of intensive care clinicians with published guidelines or the implementation of protocols to promote light sedation [10]. A meta-analysis has reported that most sedation trials failed to achieve optimal sedation, and more than two-thirds of studies reported inappropriate deep sedation [11]. Sedation practice surveys did not assess patients longitudinally and are therefore unable to draw conclusions on the incidence of deep sedation or its association with relevant outcomes [12-14].

Randomised clinical trials and observational cohort studies have largely not accounted for the early prescription of sedative agents or the early depth of sedation, in particular in the first $48 \mathrm{~h}$ after the initiation of mechanical ventilation. Early deep sedation is therefore a risk factor that may be overlooked while clinicians' attention focuses on more pressing issues and priorities. The occurrence of early deep sedation, therefore, can have a significant confounding effect on the results of sedation trials if not taken into consideration.

The Australian New Zealand sedation practice in intensive care evaluation (ANZ SPICE) study [15] revealed that early sedation depth predicts important outcomes such as mortality. To assess the strength of this relationship outside an ANZ model of care, we replicated the ANZ SPICE in Malaysian ICUs. In this prospective, longitudinal, multicentre cohort study, we assessed current sedation practice and the prevalence of deep sedation, particularly early after initiation of mechanical ventilation, and quantified the relationship between early deep sedation and important clinical outcomes including time to extubation, delirium and hospital and 180-day mortality.

\section{Materials and methods}

Study design and process

We conducted a multicentre, prospective, longitudinal, observational, non-interventional cohort study in 11 ICUs (8 public, 2 university and 1 tertiary specialist centres) in Malaysia from March to July 2011 using the ANZ SPICE protocol [15]. All participating ICUs were managed by full-time intensivists and/or anaesthesiologists with a 24/7 on-duty senior medical officer. In ventilated patients, the nurse:patient ratio was 1:2 in most ICUs. The study was approved by The National Medical Research Register and consent waived by the institutional ethics committee at participating sites.

The study was conducted in collaboration with the ANZ Intensive Care Research Centre (ANZIC RC) and the Monash University Centre for Clinical Research Excellence in Therapeutics. A streamlined case report form with Optical Recognition Software was used and sent to the ANZIC RC for data entry, data queries and analysis.

\section{Inclusion and exclusion criteria}

Patients were included if they had been ventilated within the previous $24 \mathrm{~h}$, were receiving continuous or intermittent sedative and/or analgesic medication and were expected to be ventilated for longer than $24 \mathrm{~h}$. Patients were excluded if they were $<18$ years, had proven or suspected neurological impairment, psychiatric illness, burns, dementia, palliative care or were unable to communicate with carers/investigators because of language difficulty. No centre recruited more than 30 patients. 


\section{Study logistics}

Principal investigators, research staff and senior nurses at each participating centre were trained in the study procedures, including bedside twin assessment of patients using the Richmond Agitation and Sedation Scale (RASS) [16] and the confusion assessment method for intensive care (CAM-ICU) [17]. Assessors were allowed to use local dialects (English, Malay, Hindi, Mandarin or Cantonese) to communicate with patients. To conduct the inattention (feature II) part of the CAM-ICU in patients who had difficulty with the alphabet, numbers were used (ten random numbers where the number 3 was repeated four times) instead of the (SAVEAHAART) phrase [18]. CAM-ICU was conducted ONLY if patients were in a RASS range of -2 to +1 (lightly sedated).

Definitions and data collection methods

Relevant baseline demographic data including hospital and ICU admission details and Acute Physiology and Chronic Health Evaluation II (24-h APACHE II) scores were collected [19].

Patients were followed from ICU admission to ICU discharge or 28 days in the ICU, which ever came first. The first $48 \mathrm{~h}$ following initiation of mechanical ventilation was considered "early" and $>48 \mathrm{~h}$ the "subsequent" period. RASS was assessed every $4 \mathrm{~h}$ and CAM-ICU assessed daily by trained nurses or principal investigators. Patients in a RASS range of -2 to +1 were considered lightly sedated, RASS range of -3 to -5 deeply sedated and $>2$ agitated. Patients were considered delirious if the CAM-ICU was positive. Patients were considered coma and delirium free if they had a RASS above -3 with a negative CAM-ICU score. Administration details and dosage of all sedative, analgesic and adjunct medications (intravenous infusion or bolus) were collected daily. Survival status at ICU and hospital discharge and at 180 days was collected. Only patients who reside in Malaysia were followed for 180-day mortality.

\section{Statistical analysis}

The statistical analysis was modelled on that used in the ANZ SPICE [15] and independently conducted at the ANZIC RC, Monash University, Centre for Clinical Research Excellence in Therapeutics.

\section{Sample size}

Based on the ANZIC SPICE data, 260 patients would have $90 \%$ power with a two-sided $p$ value of 0.05 to detect a difference of 2 in the number of times deep sedation was reported in the first $48 \mathrm{~h}$ between patients who died or survived. This would also allow $80 \%$ power to identify point estimates within $5 \%$ of the true value of an event assuming it occurred in $10 \%$ of patients.

\section{Statistical analysis}

All patients were included in the analysis. Comparisons of proportions were performed using chi-square tests for equal proportions or Fisher's exact tests where appropriate. Normally distributed continuous variables were compared using Student's t tests. Non-normally distributed variables were compared using Wilcoxon rank-sum tests.

RASS scores were treated as ordinal data; however, the RASS range of deep sedation $(-3$ to -5$)$ was considered a continuous variable with every additional RASS level in the deep sedation range adding to the intensity of sedation depth. Early deep sedation was the primary exposure variable in the time to event analysis of outcomes occurring after $48 \mathrm{~h}$ : time to extubation, time to subsequent delirium, time to hospital death and 180-day mortality. Time to event analysis was performed using Cox proportional hazard regression and reported as hazard ratios (95\% CI) and as Kaplan-Meier curves with a corresponding log-rank test. All patients with an ICU stay greater than $48 \mathrm{~h}$ were included in the Kaplan-Meier estimates with conventional censoring at the time of death. Adjustment of observed effects due to known or suspected potential confounders was undertaken using multivariable analysis using a list of a priori defined covariates comprising APACHE III diagnosis (cardiac, respiratory, gastrointestinal, sepsis or other), age, sex, APACHE II score, operative admission (surgical), elective admission and the cumulative doses of midazolam and dexmedetomidine, and the use of vasopressors and dialysis within the first $48 \mathrm{~h}$ of admission. All patients with ICU lengths of stay less than $48 \mathrm{~h}$ were excluded from the multivariate analysis. A two-sided $p$ value of 0.05 was considered to be statistically significant. Analysis was performed using SAS version 9.2 (SAS Institute Inc., Cary, NC, USA) and STATA version 11.0 (StataCorp LP College Station, TX, USA).

\section{Results}

Patients' demographics and baseline characteristics

We enrolled 259 patients; the main source of admission was from the general ward and emergency department (166) $64 \%$, with only (35) $13.5 \%$ of patients admitted after elective surgery. Mortality at 6 months was $46.4 \%$; loss to follow-up occurred in (22) $8.5 \%$ patients. Baseline demographics and clinical characteristics for the 
Table 1 Patients' demographics and clinical characteristics

\begin{tabular}{|c|c|c|c|c|}
\hline \multirow[t]{2}{*}{ Patients' characteristics } & \multirow{2}{*}{$\begin{array}{l}\text { Primary cohort } \\
(n=259)\end{array}$} & \multicolumn{3}{|c|}{ Divided by sedation level at $48 \mathrm{~h}$ in patients with ICU stay $>48 \mathrm{~h}$} \\
\hline & & $\begin{array}{l}\text { Lightly sedated } \\
(n=45)\end{array}$ & $\begin{array}{l}\text { Deeply sedated } \\
(n=209)\end{array}$ & $P$ value \\
\hline Age, mean (SD), years & $53.1 \pm 15.9$ & $53.6 \pm 13.9$ & $53.2 \pm 16.3$ & 0.87 \\
\hline Male $(n) \%$ & (162) $62.5 \%$ & (25) $55.5 \%$ & (134) $64.1 \%$ & 0.28 \\
\hline Weight, mean (SD), kg & $68.2 \pm 19.8$ & $72.7 \pm 22.2$ & $67.4 \pm 19.4$ & 0.10 \\
\hline APACHE II score, mean (SD) & $21.3 \pm 8.2$ & $20.1 \pm 7.8$ & $21.6 \pm 8.3$ & 0.24 \\
\hline Operative admission diagnosis $(n) \%$ & (83) $32.0 \%$ & (18) $40.7 \%$ & (65) $31.1 \%$ & 0.25 \\
\hline Respiratory failure admission diagnosis ${ }^{\mathrm{a}}(n) \%$ & (66) $25.5 \%$ & (13) $28.9 \%$ & (51) $24.4 \%$ & 0.53 \\
\hline Cardiovascular admission diagnosis ${ }^{\mathrm{a}}(n) \%$ & (39) $15.4 \%$ & (11) $24.4 \%$ & (29) $13.9 \%$ & 0.08 \\
\hline Vasopressors infusions $(n) \%$ & (209) $80.7 \%$ & (28) $62.2 \%$ & (164) $78.4 \%$ & 0.021 \\
\hline Renal replacement therapy $(n) \%$ & (73) $28.2 \%$ & (7) $15.6 \%$ & (43) $20.6 \%$ & 0.44 \\
\hline Tracheostomy after ICU admission $(n) \%$ & (36) $14 \%$ & (7) $15.6 \%$ & (28) $13.4 \%$ & 0.71 \\
\hline Time to tracheostomy, median (IQR) days & $9.35(5.63-15.34)$ & $11(5.67-16)$ & $9.35(5.63-13.7)$ & 0.86 \\
\hline Ventilation days, median (IQR) & $4.96(2.99-8.8)$ & $3.95(2.7-6.9)$ & $6.69(4-11.7)$ & 0.008 \\
\hline 28-Day coma and delirum-free days & $24(0-26)$ & $28(26-28)$ & $23(0-26)$ & $<0.0001$ \\
\hline ICU length of stay, median (IQR) days & $7.19(4.8-11)$ & $6.52(4.83-6.69)$ & $7.7(4.95-11.57)$ & 0.35 \\
\hline Hospital length of stay, median (IQR) days & $16.4(10.0-30.7)$ & $17.6(12.7-32.2)$ & $16.5(9.5-28.9)$ & 0.08 \\
\hline ICU mortality $(n) \%$ & (66) $25.5 \%$ & (3) $6.7 \%$ & (63) $30.1 \%$ & 0.001 \\
\hline Hospital mortality $(n) \%$ & (82) $31.7 \%$ & (6) $13.4 \%$ & (76) $36.4 \%$ & 0.003 \\
\hline 180-day mortality $(n) \%$ & (110/237) $46.4 \%$ & (10/41) $24.4 \%$ & (100/192) $52.1 \%$ & 0.001 \\
\hline
\end{tabular}

$n$ Number, $S D$ standard deviation, IQR interquartile range, APACHE II Acute Physiologic Assessment and Chronic Health Evaluation a APACHE III diagnostic codes [19]

primary cohort and for patients who were lightly sedated versus those who were deeply sedated at $48 \mathrm{~h}$ are shown in Table 1.

Sedative and analgesic prescription

\section{Overall therapy}

Patients were followed up to a total of 2,657 ICU days, of which $72.8 \%(1,935)$ were ventilated days and $91.8 \%$ $(2,439)$ days in which sedative and/or analgesic medications were administered. Midazolam was the predominant sedative agent given to $93 \%$ (241) of patients on $39.6 \%$ (966) of study days. Propofol prescription was significantly less and only given to $74(28 \%)(P<0.0001$ compared with midazolam) of patients on $7.5 \%$ (183) of study days. The primary analgesics used were morphine and fentanyl in $77.6 \%$ (199) and $60.2 \%$ (156) $(P<0.001)$ of patients respectively. Dexmedetomidine was given to $29.3 \%$ (76) of patients. Adjunct medications such as haloperidol, diazepam and ketamine were uncommonly used in $8.9 \%$ (21), $2.3 \%$ (6) and $5.8 \%$ (14) of patients respectively.

\section{Prescription over first $48 \mathrm{~h}$}

In the $48 \mathrm{~h}$ following initiation of mechanical ventilation, most patients received midazolam [88.4\% (229)] and/or morphine $[71.4 \%$ (185)] and/or fentanyl [40.5\%(105)]. Dexmedetomidine and propofol were given to $16.2 \%$
(42) and $10 \%$ (26) of patients respectively. Table 2 shows the sedative, analgesic and adjunct drug administration in the first $48 \mathrm{~h}$ and throughout the study period including cumulative per patient, per day dosage and the number of prescription days for each agent.

Early and subsequent sedation level

Over a total of 2,657 ICU study days, 13,836 RASS assessments were made. At first assessment, $71 \%$ (182/ 257) of patients were in the deep sedation range (RASS -3 to -5 ). Of all RASS assessments conducted early (first $48 \mathrm{~h}$ ), $58 \%(1,658)$ were in the deep sedation range, $39.3 \%(1,124)$ in the light sedation range $(-2$ to +1$)$ and $2.8 \%$ (80) in the RASS range of 2-4. During the first $48 \mathrm{~h}$ after initiation of mechanical ventilation, the median (IQR) of all RASS scores was $-3[-4-(-1)]$. At the end of $48 \mathrm{~h}, 61 \%$ (159) of patients were still deeply sedated.

After $48 \mathrm{~h}$ and subsequently throughout the study period, the median (IQR) RASS scores were -1 [ $-3-0]$, with $34 \%(4,528 / 13,319)$ of RASS assessments in the deep sedation range, $62.4 \%(8,311 / 13,319)$ in the lightly sedated range, and only $3.5 \%(466 / 13,319)$ RASS assessments were between 2 and 4 . There was a significant difference in the intensity of sedation scored in the first $48 \mathrm{~h}$ compared with subsequent study days (Fig. 1).

The main reported indications for deep sedations were controlled ventilation $64.9 \%(288 / 444)$ and concurrent muscle relaxation and severe agitation $35.1 \%(156 / 444)$ of days where an indication for deep sedation was present. Clinicians prescribed a sedation target on $22.8 \%$ 
Table 2 Sedative, analgesic and adjunct medications given

\begin{tabular}{|c|c|c|c|c|c|}
\hline \multirow[t]{2}{*}{ Drugs given } & \multicolumn{2}{|c|}{ During the first $48 \mathrm{~h}$} & \multicolumn{3}{|c|}{ During entire study } \\
\hline & $\begin{array}{l}\text { ICU } \\
\text { days } \\
518 n(\%)\end{array}$ & $\begin{array}{l}\text { Dose }^{\mathrm{a}} \\
\left.\text { (patient }^{-1} \text { day }^{-1}\right)\end{array}$ & $\begin{array}{l}\text { ICU } \\
\text { days } \\
2,439 n(\%)\end{array}$ & $\begin{array}{l}\operatorname{Dose}^{\mathrm{a}} \\
\left(\text { patient }^{-1} \text { day }^{-1}\right)\end{array}$ & $\begin{array}{l}\text { Duration treatment } \\
\text { days }{ }^{b}\end{array}$ \\
\hline Midazolam (mg) & $389(75.1 \%)$ & $28(12-58)$ & $966(39.6 \%)$ & $31(12-66)$ & $3.37(3.4)$ \\
\hline Propofol (mg) & $36(6.9 \%)$ & $240(100-680)$ & $183(7.5 \%)$ & $350(100-800)$ & $0.71(1.4)$ \\
\hline Morphine (mg) & $327(63.1 \%)$ & $24.5(12.54)$ & $773(31.7 \%)$ & $25(10-51)$ & $2.99(3.23)$ \\
\hline Fentanyl & $168(32.4 \%)$ & $290(95-620)$ & $771(31.6 \%)$ & $450(200-780)$ & 2.97 (4.19) \\
\hline Dexmedetomidine & $65(12.5 \%)$ & $1.93(0.9-4.3)$ & $334(13.7 \%)$ & $3.96(1.98-7.2)$ & $1.29(2.67)$ \\
\hline Ketamine (mg) & $16(3.1 \%)$ & $390(157-750)$ & $46(1.9 \%)$ & $1,374(315-3,840)^{\mathrm{c}}$ & $0.18(0.84)$ \\
\hline Haloperidol (mg) & $6(1.2 \%)$ & $7.5(2.2-13.5)$ & $51(2.1 \%)$ & $15(5-46)^{\mathrm{c}}$ & $0.19(0.79)$ \\
\hline Diazepam (mg) & $2(0.4 \%)$ & $4.2(2.2-6)$ & $20(0.8 \%)$ & $21(10-15)^{\mathrm{c}}$ & $0.08(0.69)$ \\
\hline
\end{tabular}

${ }^{\mathrm{a}}$ Cumulative median (IQR) dose mg (when stated), $\mu \mathrm{g}$ (dexmedetomidine and fentanyl) patient ${ }^{-1} \mathrm{day}^{-1}$

${ }^{b}$ Mean (SD)

${ }^{c}$ Cumulative dose patient ${ }^{-1}$

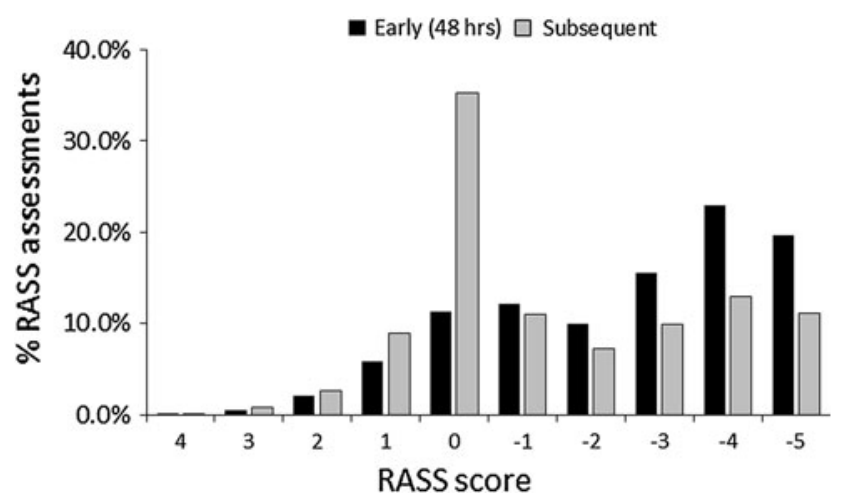

Fig. 1 RASS assessments during early (first 48 h) and subsequent study days. During the first $48 \mathrm{~h}$ following initiation of mechanical ventilation, 2,859 RASS assessments were conducted, of which $58 \%(1,658)$ were in the -3 to -5 range compared to $34 \%(4,258 /$ $13,319)$ in the -2 to +1 range $(P<0.0001)$. There was a significant increase in the RASS score of $0(\mathrm{Calm})$ after the first $48 \mathrm{~h}[(4,688 / 13,319) 35.2 \%$ versus $(323) 11.3 \%(P<0.0001)$ in the first $48 \mathrm{~h}]$

$(3,074 / 13,481)$ of occasions, and the target was achieved in $47 \%(1,446)$ of assessments. Deliberate cessation of all sedatives and analgesics occurred on $20 \%(485 / 24,240)$ of study days; however, routine daily sedation interruption was rarely implemented in only $2.3 \%$ (56) of days.

Throughout the study period, neuromuscular blockers were used on $4.1 \%$ (100) of ICU days, while physical restraints were used on $21.1 \%$ (515) of days. Self extubation was recorded in $0.4 \%$ (10) of episodes.

\section{Occurrence of delirium}

Delirium (positive CAM-ICU during RASS -2 to +1 ) was reported in $27.4 \%(20 / 73)$ of patients at first assessment and in $44 \%$ (114) of patients throughout the study period for a median (IQR) of 2 (1-4) days. In patients who stayed $>8$ days in the ICU, delirium was present in $50 \%(33 / 66)$ and $68.9 \%(31 / 45)$ of patients who stayed in the ICU longer than 14 days. Coma and delirium-free days at 28 days were significantly more in patients lightly sedated in the first $48 \mathrm{~h}$ (Table 1).

Early sedation depth and clinical outcomes

Kaplan-Meier estimates showed that the patients who were deeply sedated at $48 \mathrm{~h}$ had a significantly longer time to extubation (log-rank $P=0.008$ ) (Fig. 2) and higher hospital (log-rank $P=0.004$ ) and 180-day mortality (log-rank $P=0.001$ ) (Fig. 3). Univariate analysis showed that time to delirium after $48 \mathrm{~h}$ was also significantly shorter with early deep sedation (OR $0.6495 \%$ CI $0.57-0.73, P<0.001)$.

Multivariable Cox proportional hazard regression, adjusted for relevant covariates (Table 3), showed that the occurrence of every additional RASS in the -3 to -5 range was independently associated with a reduced chance of shorter time to extubation (HR 0.93, $95 \% \mathrm{CI}$ $0.89-0.97, P=0.0001$ ), hospital death (HR 1.11, $95 \%$ CI $1.04-1.18, P<0.001)$ and 180 -day mortality (HR $1.10,95 \%$ CI $1.04-1.15, P=0.002)$. Trend analysis using the Cochran-Mantel-Haenszel test showed that these associations were approximately linear (0.03). Early deep sedation was not associated with time to delirium occurring after $48 \mathrm{~h}$ (HR 0.98, $P=0.46$ ).

Multivariable analysis with hospital mortality showed that early deep sedation (HR 1.13, $95 \%$ CI 1.06-1.20, $P<0.0001$ ), APACHE II (HR 1.04, $95 \%$ CI 1.00-1.08, $P=0.04$ ) and a cardiac admission diagnosis (HR 3.51, $95 \%$ CI 1.31-9.44, $P=0.01$ ) were associated with hospital mortality.

Adjusted multivariable analysis also showed that the cumulative dose of midazolam in the first $48 \mathrm{~h}$ was associated with the RASS -3 to -5 , but it was not 
Fig. 2 Kaplan-Meier curves for time to extubation. Time to extubation was significantly longer amongst patients who were deeply sedated early compared with those that were not. Median (IQR) time to extubation was 3.95 (2.7-6.9) rank $P<0.008)$

Fig. 3 Kaplan-Meier curves for 180-day mortality. Those who were deeply sedated early (first $48 \mathrm{~h}$ ) showed a significant increase in risk of death at 6 months (log-rank $P=0.001)$ compared with patients who were not deeply sedated versus $6.69(4-11.7)$ days $(\log$ -

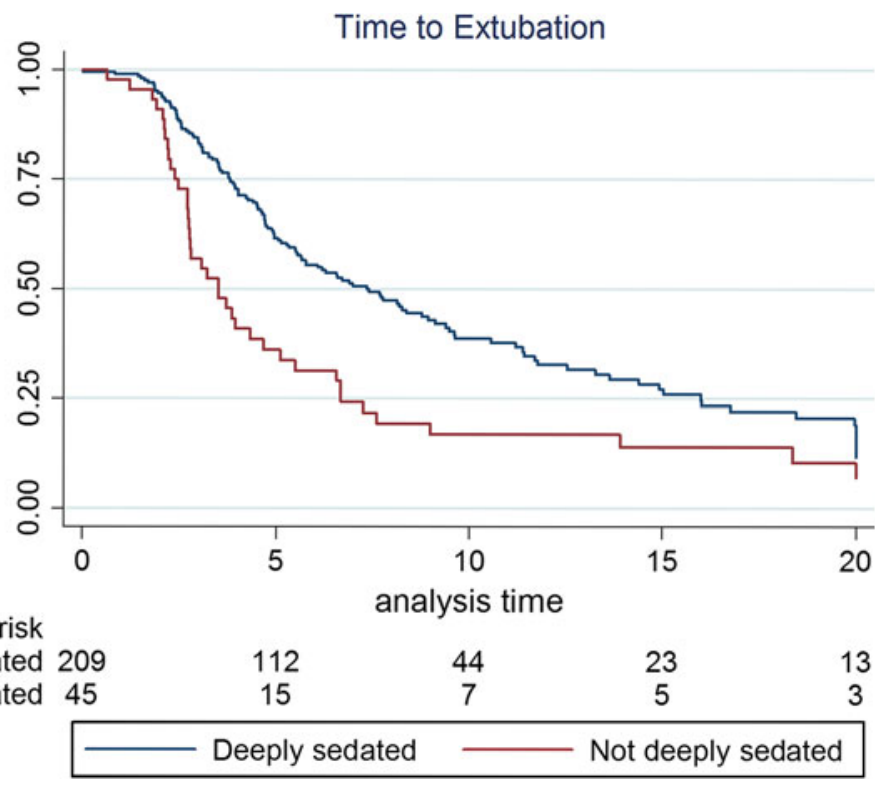

\begin{tabular}{rccccc} 
Number at risk & \multicolumn{4}{c}{ analysis time } \\
Deeply sedated & 209 & 112 & 44 & 23 & 13 \\
Not deeply sedated & 45 & 15 & 7 & 5 & 3 \\
\cline { 2 - 5 } \cline { 5 - 5 } & & Deeply sedated & & Not deeply sedated \\
\cline { 2 - 5 } & & &
\end{tabular}

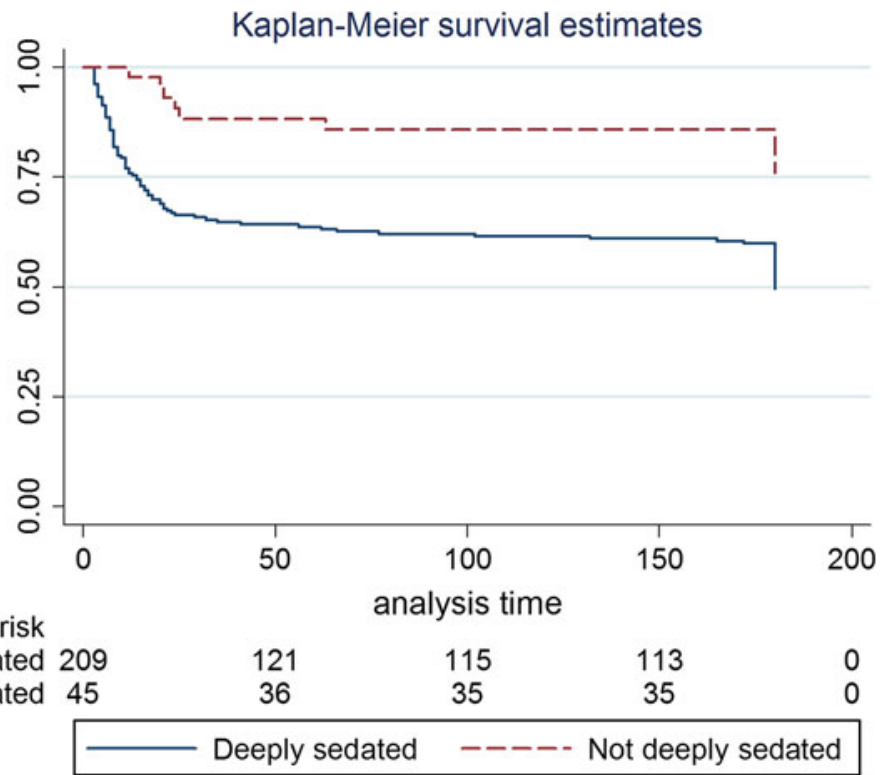

associated with time to extubation, delirium or death. The cumulative dose of dexmedetomidine in the first $48 \mathrm{~h}$, however, was significantly associated with delayed time to delirium occurring after $48 \mathrm{~h}$ (HR $1.4195 \% \mathrm{CI}$ $1.13-1.76, P=0.002)$.

\begin{tabular}{|c|c|c|c|c|c|}
\hline Number at risk & & \multicolumn{3}{|c|}{5} & \\
\hline Deeply sedated & 209 & 121 & 115 & 113 & 0 \\
\hline Not deeply sedated & 45 & 36 & 35 & 35 & 0 \\
\hline
\end{tabular}

after initiation of mechanical ventilation and important clinical outcomes. Early deep sedation was independently associated with time to extubation and mortality. The occurrence of every additional RASS assessment in the deep sedation range was associated with a $13 \%$ increase in the risk of death at hospital discharge (and $10 \%$ at 6 months) and a $7 \%$ delay in extubation ( $8.5 \mathrm{~h})$. All these associations remained significant after adjusting for multiple covariates including sedative choice, admission diagnosis and severity of illness.

We also found that midazolam and morphine were the most commonly used agents for sedation and analgesia in We identified a significant independent relationship Malaysian ICUs for patients ventilated for longer than
between the intensity of sedation in the first $48 \mathrm{~h}$ (early) $24 \mathrm{~h}$, and the use of propofol was less frequent. 
Table 3 Multivariable proportional hazard Cox regression of time to extubation, delirium and 180-day mortality versus early sedation depth (RASS -3 to -5 ) as primary exposure variable

\begin{tabular}{|c|c|c|c|c|c|c|c|c|c|}
\hline & \multicolumn{3}{|c|}{ Time to extubation } & \multicolumn{3}{|c|}{ Delirium after $48 \mathrm{~h}$} & \multicolumn{3}{|c|}{ 180-Day mortality } \\
\hline & HR & $95 \% \mathrm{CI}$ & $P$ & HR & $95 \%$ CI & $P$ & HR & $95 \%$ CI & $P$ \\
\hline RASS -3 to $-5^{\mathrm{a}}$ & 0.93 & $0.89-0.96$ & 0.0001 & 0.98 & $0.94-1.03$ & 0.46 & 1.10 & $1.04-1.15$ & 0.0002 \\
\hline APACHE II ${ }^{\mathrm{b}}$ & 0.96 & $0.94-0.98$ & 0.001 & 0.98 & $0.96-1.01$ & 0.22 & 1.02 & $0.10-1.05$ & 0.08 \\
\hline Age & 1.00 & $0.99-1.01$ & 0.77 & 1.01 & $1.00-1.03$ & 0.04 & 1.02 & $1.00-1.03$ & 0.01 \\
\hline Male & 0.84 & $0.61-1.16$ & 0.29 & 1.37 & $0.92-2.04$ & 0.12 & 0.75 & $0.50-1.14$ & 0.17 \\
\hline Operative & 1.22 & $0.74-2.04$ & 0.43 & 1.10 & $0.60-1.03$ & 0.75 & 0.56 & $0.26-1.21$ & 0.14 \\
\hline Elective & 0.99 & $0.54-1.79$ & 0.96 & 1.31 & $0.65-2.64$ & 0.44 & 0.83 & $0.32-2.13$ & 0.70 \\
\hline Cardiac $^{c}$ & 0.84 & $0.47-1.50$ & 0.56 & 1.17 & $0.59-2.32$ & 0.66 & 2.22 & $0.92-5.36$ & 0.07 \\
\hline Respiratory $^{\mathrm{c}}$ & 0.82 & $0.51-1.31$ & 0.41 & 0.94 & $0.51-1.73$ & 0.84 & 1.14 & $0.57-2.29$ & 0.71 \\
\hline Sepsis ${ }^{c}$ & 0.97 & $0.59-1.60$ & 0.91 & 1.22 & $0.65-2.31$ & 0.54 & 1.22 & $0.59-2.50$ & 0.60 \\
\hline Gastrointes $^{c}$ & 0.61 & $0.33-1.12$ & 0.11 & 0.94 & $0.46-1.90$ & 0.86 & 2.42 & $1.02-5.75$ & 0.04 \\
\hline Vasopressors & 1.09 & $0.73-1.64$ & 0.67 & 1.15 & $0.70-1.94$ & 0.54 & 1.42 & $0.81-2.47$ & 0.22 \\
\hline Dialysis $^{\mathrm{d}}$ & 0.53 & $0.32-0.86$ & 0.01 & 1.03 & $0.60-1.76$ & 0.92 & 0.94 & $0.94-2.45$ & 0.09 \\
\hline
\end{tabular}

${ }^{\mathrm{a}}$ For every additional RASS in deep sedation, chance of achieving the desired outcome (shorter time to extubation) was reduced by $7 \%(4-11 \%)$

b Acute Physiologic Assessment and Chronic Health Evaluation II

Other studies on sedation-related outcomes

Our findings are consistent with findings of the ANZ SPICE study [15] cohort and lend strong support to the notion that early deep sedation is an important and potentially modifiable risk factor for delayed time to extubation and increased mortality. Furthermore, our results suggest that these findings are neither confined to an ANZ ICU model of care nor determined by a particular sedative regimen. Thus, it is plausible that these findings are generalisable to a wider intensive care setting and that early deep sedation may be a global problem associated with poor outcome.

Patients enrolled in this cohort have similar characteristics to patients recruited in the ANZ SPICE study [15] and in other observational [20] and interventional studies $[21,22]$ with a high APACHE II score and primarily medical diagnoses with standard ICU interventions. The intensity of sedation depth reported at enrolment, early and throughout the study period is comparable to that reported in the ANZ SPICE [15] study. All of this provides external validity and confidence that our findings may be applicable to similar cohorts.

Randomised trials of sedation practice have largely ignored this early period following initiation of mechanical ventilation and do not account for sedation practice and sedation depth prior to randomisation. In the largest sedation trials published so far, including the most recent randomised twin trials of sedation [23, 24], patients were randomised up to 72-96 h after ICU admission, potentially limiting the full treatment effect of the interventions tested.

Our study is the first to report the incidence of delirium in Malaysian ICUs. Although the CAM-ICU was only assessed during light sedation, $44 \%$ of patients developed an episode of delirium. It is possible that c APACHE III admission diagnostic codes

d Vasopressors and any form of dialysis within the first $48 \mathrm{~h}$

delirium was underdiagnosed in our cohort; however, our patients were on average a few years younger than those in ANZ SPICE, inferring a lower risk of delirium. Although reduced sensitivity of the CAM-ICU has been reported [25, 26], delirium was methodologically and diligently assessed in our cohort by trained research and study staff. While sedative induced coma is believed to increase the risk of delirium [27], we did not find early deep sedation or the cumulative dose of sedative agents to independently predict time to delirium. Dexmedetomidine given in the first $48 \mathrm{~h}$, however, independently predicted delayed time to delirium occurring after $48 \mathrm{~h}$.

\section{Studies of sedation depth}

Reducing overall deep sedation has been shown to improve a variety of clinical outcomes. Strategies to reduce sedation depth included sedation interruption [5], protocolised sedation $[6,8,28]$ and analgo-sedation [7]. All appear to lead to shorter ventilation time, attenuation of delirium and trend to reduced mortality. It is important, however, to implement strategies to reduce sedation depth early after initiation of mechanical ventilation. This would be more challenging in the context of a randomised clinical trial.

The practice of daily sedation interruption was not common in our cohort. Multiple reports [21, 22, 29] questioned the utility of routine sedation interruption where sedation algorithms are routinely used.

Our study supports the need for an adequately powered process of care sedation trial that addresses the problem of early deep sedation. This can be achieved through early delivery of interventions, which are likely to reduce the intensity of early and subsequent sedation while providing comfort and safety to patients. Such interventions may 
combine the use of agents known to promote light cooperative sedation such as propofol [30] or dexmedetomidine $[23,24]$ with targeted light sedation. Importantly, future trials should assess long-term patient-centred outcomes such as mortality and cognitive function as the main outcomes.

\section{Strengths and limitations}

The primary strengths of our study were the detailed and comprehensive assessment of patients from ICU admission (including the crucial first $48 \mathrm{~h}$ ) to 28 days and an 180-day follow-up. This is the first study of this nature to be conducted outside the developed world; however, the study was supported by an experienced research methods centre and centrally coordinated and monitored; thus, the data quality was high. Robust statistical methods assessing time-to-event analysis using multivariate Cox proportional hazard regression adjusting for a priori selected covariates were used. Cohort studies, however, cannot establish causation, and unmeasured confounders could not be adjusted for in the statistical model.

\section{Conclusion}

In mechanically ventilated critically ill patients with mainly medical ICU admission diagnoses, the intensity of sedation in the first $48 \mathrm{~h}$ after initiation of mechanical ventilation was independently associated with time to extubation and mortality. These findings strengthen the premise that early sedation depth is a potentially modifiable target to improve important clinical outcomes and a universal problem in intensive care sedation worldwide. Future sedation trials should account for the choice of sedative agents and sedative delivery shortly after initiation of mechanical ventilation.

Acknowledgments We are grateful for the assistance rendered by support staff, nurses and doctors in the participating centres. We thank Ms. Norfazlina Jaffar at the National Heart Institute, Kuala Lumpur. We thank Ms. Belinda Howe at the Australian New
Zealand Intensive Care Research Centre for study coordination and the staff of the Clinical Informatics and Data Management Unit CCRET, Monash University. The project received funding from the National Heart Institute Foundation Kuala Lumpur, Malaysia.

Conflicts of interest No relevant conflicts of interest are declared.

Open Access This article is distributed under the terms of the Creative Commons Attribution Noncommercial License which permits any noncommercial use, distribution, and reproduction in any medium, provided the original author(s) and the source are credited.

\section{Appendix}

Participating centers and co-investigators

University of Malaya: Dr. KK Wong, Dr. Suresh Venugopal, Dr. Vineya Rai, Dr. Mohd Shahnaz, Vimala Ramoo (Nurse Lecturer). National Heart Institute: Dr. Smitha Jose, Dr. Ozlem Ozturk, SN Zuraida Ramlee, Staff Nurse Bong Siu Foon, Staff Nurse Rohana Amran. Hospital Melaka: Dr. R.K. Anusha Narula, Dr. Erin Shazrin Md Ramly, Dr. Khalidah Abdul Hapiz, Dr. Lim I-Liang, Dr. Mohamad Hafiz Che Morad. Hospital Raja Perempuan Zainab II: Dr. Mohd Nazri Ali, Dr. H. Noor Raihan, Sister I. Azizum, Staff Nurse Y. Suzana, Staff Nurse H. Haryati. Kuala Lumpur General Hopsital: Staff Nurse S. Salmi Zawati, Staff Nurse J. Nur Ismee. Hospital Queen Elizebeth: Dr. Mohd Ashraf Zulkarnain. Hospital University Sains Malaysia: Assoc. Prof. Dr. Mahamarowi Omar, Dr. Siti Aisah Omar, Sister Rokian Ismail, Staff Nurse Norhamilah Hassan, Staff Nurse Zanariah Zakaria. Sarawak General Hospital: Dr. Sanah Mohtar, Dr. Marina Ahmad, Staff Nurse Winnie Suai, Staff Nurse Wong Ai Li, Staff Nurse Jong Siaw Lan. Hospital Sultanah Bahiyah: Dr. S. Siti Rohayah. Dr. Fitriah Mahadir, Staff Nurse Teoh Shook Lian, Staff Nurse Maryam Md Zain, Staff Nurse Noorasmah Ahmad. Hospital Sultanah Aminah: Dr. K. Mahazir, Staff Nurse A'ishah Abu Bakar. Penang General Hospital: Dr. Ho Wing Nan, Sister Tan Ai Ping, Sister Chin Lai Ngan, Dr. Lim Chiew Har, Dato Dr. Jahizah Hassan.

\section{References}

1. Jacobi J, Fraser GL, Coursin DB et al (2002) Clinical practice guidelines for sustained use of sedatives and analgesics in the critically ill adults: Task Force of the American College of Critically Care Medicine (ACCM) of Society of Critical Care Medicine (SCCM), American Society of HealthSystem Pharmacists (ASHP), American College of Chest Physicians. Crit Care Med 30:119-141
2. Payen JF, Chanques G, Mantz J et al (2007) Current practices in sedation and analgesia for mechanically ventilated critically ill patients: a prospective multicenter patient-based study. Anesthesiology 106:687-695

3. Devlin JW (2008) The pharmacology of over sedation in mechanically ventilated adults. Curr Opin Crit Care 14:403-407
4. Foster J (2005) Complications of sedation and critical illness. Crit Care Nurs Clin North Am 17:287-296

5. Kress JP, Pohlman AS, O'Connor MF et al (2000) Daily interruption of sedative infusions in critically ill patients undergoing mechanical ventilation. N Engl J Med 342:1471-1477 
6. Skrobik Y, Ahern S, Leblanc M, Marquis F, Awissi DK, Kavanagh BP (2010) Protocolized intensive care unit management of analgesia, sedation, and delirium improves analgesia and subsyndromal delirium rates. Anesth Analg 111:451-463

7. Strøm T, Martinussen T, Toft P (2010) A protocol of no sedation for critically ill patients receiving mechanical ventilation: a randomised trial. Lancet 375:475-480

8. Teggiari MM, Romand JA, Yanez ND et al (2009) Randomized trial of light versus deep sedation on mental health after critical illness. Crit Care Med 37:2527-2534

9. Patel SB, Kress JP (2012) Sedation and Analgesia in the mechanically ventilated patient. Am J Respir Crit Care Med 185:486-497

10. Mehta S, McCullagh I, Burry L (2009) Current sedation practices: lessons learned from international surveys. Crit Care Clin 25:471-488

11. Jackson DL, Proudfoot CW, Cann KF, Walsh TS (2009) The incidence of suboptimal sedation in the ICU: a systematic review. Crit Care 13(6):R204

12. Shehabi Y, Botha JA, Boyle MS, Ernest D, Freebairn RC, Jenkins IR, Roberts BL, Seppelt IM (2008) Sedation and delirium in the intensive care unit: an Australian and New Zealand perspective. Anaesth Inten Care. 36:570-578

13. Martin J, Franck M, Sigel S, Weiss M, Spies C (2007) Changes in sedation management in German intensive care units between 2002 and 2006: a national follow up survey. Crit Care 11:R124. doi:10.1186/cc6189

14. Reschreiter H, Maiden M, Kapila A (2008) Sedation practice in the intensive care unit: a UK national survey. Crit Care 12:R152. doi: 10.1186/cc7141

15. Shehabi Y, Bellomo R, Reade $M$ et al (2012) for Sedation Practice in Intensive Care Evaluation (SPICE) Study Group and the ANZICS CTG: early intensive care sedation predicts long-term mortality in mechanically ventilated critically ill patients. Am J Respir Crit Care Med. doi: 10.1164/rccm.201203-0522OC
16. Sessler CN, Gosnell MS, Grap MJ, Brophy GM, O'Neal PV, Keane KA, Tesoro EP, Elswick RK (2002) The Richmond Agitation-Sedation Scale: validity and reliability in adult intensive care unit patients. Am J Respir Crit Care Med 166:1338-1344

17. Ely EW, Inouye SK, Bernard GR, Gordon S, Francis J, May L, Truman B, Speroff T, Gautam S, Margolin R, Hart RP, Dittus R (2001) Delirium in mechanically ventilated patients: validity and reliability of the confusion assessment method for the intensive care unit (CAM-ICU). JAMA 286:2703-2710

18. Ely EW, Bun BT (2012) Delirium and CAM-ICU resources. http://www.mc. vanderbilt.edu/icudelirium/ assessment.html. Accessed Feb 2012

19. Knaus WA, Draper EA, Wagner DP, Zimmerman JE (1985) APACHE II: a severity of disease classification system. Crit Care Med 13:818-829

20. Esteban A, Anzueto A, Frutos F, Alía I, Brochard L, Stewart TE, Benito S, Epstein SK, Apezteguía C, Nightingale P, Arroliga AC, Tobin MJ (2002) Mechanical Ventilation International Study Group: characteristics and outcomes in adult patients receiving mechanical ventilation: a 28-day international study. JAMA 287:345-355

21. Mehta S, Burry L, Cook D, for the SLEAP Investigators and Canadian Critical Care Trials Group, et al (2012) SLEAP: daily sedation interruption in mechanically ventilated critically ill patients cared for with a sedation protocol. A randomized controlled trial. doi:10.1001/jama.2012.13872

22. De Wit M, Gennings C, Jenvey WI, Epstein SK (2008) Randomized trial comparing daily interruption of sedation and nursing-implemented sedation algorithm in medical intensive care unit patients. Crit Care 12:R70

23. Jakob SM, Ruokonen E, Grounds RM, Sarapohja T, Garratt C, Pocock SJ, Bratty JR, Takala J (2012) Dexmedetomidine for long-term sedation investigators dexmedetomidine versus midazolam or propofol for sedation during prolonged mechanical ventilation: two randomized controlled trials. JAMA 307:1151-1160
24. Riker RR, Shehabi Y, Bokesch PM, Ceraso D, Wisemandle W, Koura F, Whitten P, Margolis BD, Byrne DW, Ely EW, Rocha MG, SEDCOM (Safety and Efficacy of Dexmedetomidine Compared With Midazolam) Study Group (2009) Dexmedetomidine vs. midazolam for sedation of critically ill patients: a randomized trial. JAMA 301:489-499

25. Reade MC, Eastwood GM, Peck L et al (2011) Routine use of the confusion assessment method for the intensive care unit (CAM-ICU) by bedside nurses may underdiagnose delirium. Crit Care Resusc 13:217-225

26. van Eijk M, van den Boogaard M, van Marum R (2011) Routine use of the confusion assessment method for the intensive care unit: a multicenter study. Am J Respir Crit Care Med 184:340-344

27. Ouimet S, Kavanagh BP, Gottfried SB, Skrobik Y (2007) Incidence, risk factors and consequences of ICU delirium. Intensive Care Med 33:66-73

28. Girard TD, Kress JP, Fuchs BD, Thomason JW, Schweickert WD, Pun BT, Taichman DB, Dunn JG, Pohlman AS, Kinniry PA, Jackson JC, Canonico AE, Light RW, Shintani AK, Thompson JL, Gordon SM, Hall JB, Dittus RS, Bernard GR, Ely EW (2008) Efficacy and safety of a paired sedation and ventilator weaning protocol for mechanically ventilated patients in intensive care (awakening and breathing controlled trial): a randomised controlled trial. Lancet 371:126-134

29. Augustes R, Ho KM (2011) Metaanalysis of randomised controlled trials on daily sedation interruption for critically ill adult patients. Anaesth Intensive Care 39:401-409

30. Kwok MH, Joseph YN (2008) The use of propofol for medium and long-term sedation in critically ill adult patients: a meta-analysis. Intensive Care Med 34:1969-1979 